\title{
Connexins and cyclooxygenase-2 crosstalk in the expression of radiation-induced bystander effects
}

\author{
Y Zhao ${ }^{1}$, S M de Toledo ${ }^{1}, \mathrm{G} \mathrm{Hu}^{1}$, T K Hei ${ }^{2}$ and E I Azzam*,1 \\ ${ }^{1}$ Department of Radiology, Rutgers University, New Jersey Medical School, Cancer Center, Newark, NJ 07103, USA and ${ }^{2}$ Center \\ for Radiological Research, Department of Radiation Oncology, Columbia University Medical Center, New York, NY 10032, USA
}

Background: Signalling events mediated by connexins and cyclooxygenase-2 (COX-2) have important roles in bystander effects induced by ionising radiation. However, whether these proteins mediate bystander effects independently or cooperatively has not been investigated.

Methods: Bystander normal human fibroblasts were cocultured with irradiated adenocarcinoma HeLa cells in which specific connexins $(\mathrm{C} x)$ are expressed in the absence of endogenous $\mathrm{Cx}$, before and after COX-2 knockdown, to investigate DNA damage in bystander cells and their progeny.

Results: Inducible expression of gap junctions composed of connexin26 (Cx26) in irradiated HeLa cells enhanced the induction of micronuclei in bystander cells $(P<0.01)$ and reduced the coculture time necessary for manifestation of the effect. In contrast, expression of connexin32 (Cx32) conferred protective effects. COX-2 knockdown in irradiated HeLa Cx26 cells attenuated the bystander response due to connexin expression. However, COX-2 knockdown resulted in enhanced micronucleus formation in the progeny of the bystander cells $(P<0.001)$. COX-2 knockdown delayed junctional communication in HeLa Cx26 cells, and reduced, in the plasma membrane, the physical interaction of $\mathrm{C} \times 26$ with MAPKKK, a controller of the MAPK pathway that regulates COX-2 and connexin.

Conclusions: Junctional communication and COX-2 cooperatively mediate the propagation of radiation-induced non-targeted effects. Characterising the mediating events affected by both mechanisms may lead to new approaches that mitigate secondary debilitating effects of cancer radiotherapy.

Widespread evidence has challenged the paradigm that radiation traversal through the nucleus of a cell is the only prerequisite for the production of genetic damage or a biological response. In a landmark study, Nagasawa and Little reported that when $<1 \%$ of nuclei in a cell culture are traversed by an $\alpha$ particle track, more than $30 \%$ of the cells undergo an increased frequency of sisterchromatid exchanges (Nagasawa and Little, 1992). Since then, radiation-induced bystander effects (RIBE) have been demonstrated in numerous in vitro and in vivo studies using a variety of biological endpoints (reviewed in Prise and O'Sullivan, 2009). The existence of RIBE has implications for radiotherapy, diagnostic radiology and health risks of environmental radiation exposures.
Two major mechanisms mediate RIBE; in confluent cultures of normal and certain cancer cells, intercellular communication through gap junctions is a critical mediator (Azzam et al, 2001; Zhou et al, 2001), and in sub-confluent normal or tumour cell cultures, diffusible factor(s), including byproducts of cyclooxygenase activity, participate in the process (Mothersill and Seymour, 2004; Hei, 2006).

Gap junctions are intercellular channels that are expressed in virtually all human tissues. They allow small molecules, such as ions and secondary messengers, to pass freely between contiguous cells, and thus they are essential for maintaining cellular homeostasis and function (Goodenough and Revel, 1970; Willecke et al, 2002). 
They consist of connexins (Cx), which are a large family of proteins with at least 21 identified members in human cells. Junctional channels comprising specific Cx have different permeability properties (Harris, 2001). We have shown that molecules communicated through gap junctions result in the upregulation of oxidative stress in cells adjacent to those exposed to the irradiated cells (Azzam et al, 1998), and in synergistic enhancement of toxicity among the irradiated cells (Autsavapromporn et al, 2011). Regulation of connexin expression/gap junction function by chemical agents, forced connexin expression by transfection and connexin gene knockout studies provide substantial evidence for the role of gap junctions in RIBE (Azzam et al, 2001). The participation of gap junctions in toxic bystander effects is not unique to ionising radiation; it was also described in high-density cells exposed to chemotherapeutic agents (Jensen and Glazer, 2004).

Cyclooxygenase-2 (COX-2), the inducible form of cyclooxygenase, is activated by growth factors and cytokines to catalyse the conversion of arachidonic acid to prostaglandins (Subbaramaiah et al, 1998). Mitogen-activated protein kinase ( $\left.38^{\mathrm{MAPK}}\right)$ and inhibitor nuclear factor $\kappa \mathrm{B}$ kinase (IKK)-NF- $\kappa \mathrm{B}$ signalling pathways have been implicated in COX-2 upregulation. COX-2 has an important role in modulating the inflammatory response, which is often associated with genomic instability and carcinogenesis (Stasinopoulos et al, 2013). In particular, the addition of the COX-2 inhibitor NS-398 suppressed COX-2 activity and attenuated bystander mutagenesis in non-irradiated human lung fibroblasts in cultures where a small fraction of cells were traversed by $\alpha$ particles (Zhou et al, 2005). However, whether connexin channels and COX-2 mediate RIBE through common or independent mechanisms has not been investigated. Understanding the mediating events is pertinent to potentiating the efficacy of cancer radiotherapy and formulating strategies to attenuate posttherapeutic pathologies.

In this study, we used a genetic approach to examine the possible crosstalk between COX-2 and $\mathrm{Cx}$ in the propagation from irradiated tumour cells of signalling events that lead to DNA damage in contiguous bystander normal cells. We characterised micronucleus formation in bystander cells, junctional communication and connexin-interacting proteins before and after COX-2 knockdown in irradiated HeLa cells that express Cx32 or Cx26, which form channels with distinct permeability properties. We also investigated the induction of DNA damage in bystander cells in which COX-2 level was knocked down.

\section{MATERIALS AND METHODS}

Cells. AG1522 normal human diploid fibroblasts destined to be bystanders were grown in Eagles' minimum essential medium (CellGro) as described (Azzam et al, 2001). These cells express connexin26 (Cx26), connexin32 (Cx32) and connexin43 (Cx43), and functionally communicate with other cells by gap junctions and secreted factors (Azzam et al, 2003; Autsavapromporn et al, 2013). For coculture experiments, we used a Transwell insert with $1-\mu \mathrm{m}$ pores, whereby AG1522 cells destined to be bystanders were grown to the confluent state at the bottom side of the inserts. To this end, the cells were seeded on inverted inserts and allowed to attach, which occurred within $\sim 1 \mathrm{~h}$; the inserts were then reinverted and placed in 6-well plates containing growth medium. The cells were grown for 7 days with daily feeding with fresh medium. Under these conditions, $95-98 \%$ of the cells were in $\mathrm{G}_{0} / \mathrm{G}_{1}$ phase of the cell cycle when they were cocultured with tumour cells seeded on the top side of the insert.

To investigate the role of specific $\mathrm{Cx}$ in propagation of RIBE, HeLa cells expressing Cx26 or Cx32 were generated by transfecting parental cells with inducible vectors containing rat $\mathrm{Cx} 26$ or $\mathrm{Cx} 32$ cDNA. The expressed $\mathrm{Cx}$ form junctional channels that discriminate among communicated signalling molecules (Koreen et al, 2004). Cells destined for $\alpha$-particle irradiation were grown as described (Autsavapromporn et al, 2013). To induce Cx26 or Cx32 expression, the cells were incubated with $1 \mu \mathrm{g} \mathrm{ml}{ }^{-1}$ doxycycline (BD Biosciences, San Jose, CA, USA) $16 \mathrm{~h}$ before irradiation. We have shown that HeLa cells expressing Cx26 or Cx32 communicate via junctional channels with cocultured AG1522 cells grown on Transwell inserts (Autsavapromporn et al, 2013).

Irradiation. HeLa cells were exposed, at $37^{\circ} \mathrm{C}$, to $\alpha$ particles of 3.7 MeV mean energy from an ${ }^{241} \mathrm{Am}$-collimated source at a mean absorbed dose rate of $5.7 \mathrm{cGymin}^{-1}$ (Autsavapromporn et al, 2011). Immediately after irradiation, the cells were trypsinised and seeded on top of inserts having bystander AG1522 cells grown on their bottom side. After 6 or $24 \mathrm{~h}$ of coculture, the bystander cells were harvested with $99.9 \%$ purity, as assessed by a flow cytometry approach, to examine biological endpoints. In some experiments, the cells were exposed to ${ }^{137} \mathrm{Cs} \gamma$ rays at a mean absorbed dose rate of $2.75 \mathrm{~Gy} \mathrm{~min}^{-1}$ (Autsavapromporn et al, 2011).

Micronucleus formation. Micronuclei, a form of chromosomal damage that arises mainly from DNA double-strand breaks, were evaluated by the cytokinesis block technique (described in Autsavapromporn et al, 2011). At least 1000 binucleated cells/ treatment were examined in each experiment.

Inhibitors. 18- $\alpha$-glycyrrhetinic acid (AGA), a reversible inhibitor of gap junction communication, and lanthanum chloride $\left(\mathrm{La}^{3+}\right)$ (Sigma, St. Louis, MO, USA), an inhibitor of hemi-channels, were added to cell cultures at non-toxic concentrations of $50 \mu \mathrm{M} 15 \mathrm{~min}$ before coculture (Gaillard et al, 2009; Wang et al, 2013).

Western blot analysis. Total cell lysates were prepared in the presence of protease and phosphatase inhibitors. The monoclonal antibodies were as follows: anti-ERK1/2, anti-phospho-ERK1/2, anti-p38, anti-phospho-p38, anti-phospho-NF- $\kappa$ B (Cell Signaling, Beverly, MA, USA), anti-COX-2 (BD Biosciences) and antiMAPKKK (Abcam, Cambridge, MA, USA).

Immunoprecipitation. Monolayer cells were washed with PBS, and TritonX-100-insoluble fractions of plasma membrane were isolated. Immunoprecipitation with anti-Cx26 antibody and immunoblotting were according to standard techniques. Inputs (supernatants) represent $1 / 20$ th of the extract used for immunoprecipitation.

Statistical analyses. Poisson statistics was used to calculate the standard error associated with the percentage of cells with micronuclei over the total number of cells scored. The Pearson's $\chi^{2}$-test was used to compare treatment groups versus respective controls. A $P$ value of $<0.05$ was considered statistically significant. Experiments were repeated at least three times, and standard errors of the means are indicated in the figures. The data shown are from pooled experiments.

\section{RESULTS}

Gap junctions consisting of $\mathrm{Cx} 26$, but not $\mathrm{Cx} 32$, accelerate the expression of stressful RIBE. To investigate the role of gap junction permeability in RIBE, we used HeLa cells as a model system. These cells, which lack endogenous $\mathrm{Cx}$, were stably transfected with either empty vector (henceforth coined HeLa parental) or inducible vectors containing Cx26 or Cx32 cDNA (henceforth coined HeLa Cx26 or HeLa Cx32). In the transfected cells, the expressed $\mathrm{Cx}$ localise in the plasma membrane and form functional gap junctions (Koreen et al, 2004). We have previously shown that HeLa Cx26 and HeLa Cx32 cells also form functional gap junctions with normal human diploid AG1522 fibroblasts 

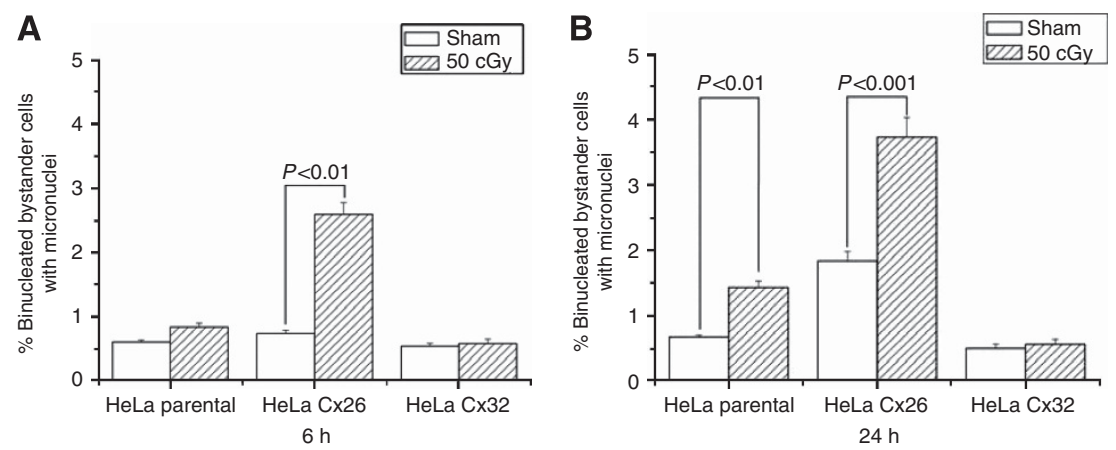

Figure 1. The effect of junctional permeability on induction of DNA damage in bystander cells. HeLa parental cells deficient in junctional communication, or expressing $\mathrm{C} \times 26$ or $\mathrm{C} \times 32$ channels, were exposed to a mean absorbed dose of 50 cGy from $3.7 \mathrm{MeV} \alpha$ particles or were shamtreated. Within 5 min after irradiation, they were cocultured with AG1522 normal cells for $6 \mathrm{~h}(\mathbf{A})$ or $24 \mathrm{~h}(\mathrm{~B})$ in a manner that permitted junctional communication between the bystander AG1522 cells and irradiated HeLa cells expressing connexins. After coculture, the bystander cells were harvested and subjected to the micronucleus formation assay.

(Autsavapromporn et al, 2013). The HeLa cells were exposed to a mean absorbed dose of $50 \mathrm{cGy}$ from $3.7 \mathrm{MeV} \alpha$ particles that results in $\sim 50 \%$ clonogenic survival (Autsavapromporn et al, 2013), and within $5 \mathrm{~min}$ after exposure the irradiated cells were intimately cocultured with bystander AG1522 cells in Transwell inserts, as described in the Methods section. After $6 \mathrm{~h}$ or $24 \mathrm{~h}$, the bystander cells were isolated and assessed for DNA damage in the form of micronuclei. Compared with coculture with sham-irradiated cells, a 6-h coculture of AG1522 cells with irradiated HeLa parental cells resulted in no significant change in the fraction of bystander cells with micronuclei (Figure 1A). However, a 24-h coculture resulted in an increase $(P<0.01)$ (Figure $1 \mathrm{~B})$. This degree of micronucleus induction in bystander cells is attributed to non-gap junctional effects (e.g., secreted factors). When the HeLa cells expressed functional Cx 26 channels, a substantial bystander effect was seen at $6 \mathrm{~h}$, and at $24 \mathrm{~h}$ of coculture the bystander effect was greater than that seen upon coculture with parental HeLa cells $(P<0.01)$ (Figure 1A). In contrast, coculture of bystander cells with irradiated HeLa Cx32 cells for $6 \mathrm{~h}$ or even $24 \mathrm{~h}$ did not induce micronucleus formation in bystander cells (Figure $1 \mathrm{~A}$ and $\mathrm{B}$ ). These data support a critical role for gap junction permeability in determining the magnitude of induced bystander effects. Whereas the expression of Cx26 in HeLa cells mediated intercellular effects leading to DNA damage in bystander normal cells, the expression of Cx32 mediated intercellular effects that mitigate the effects that otherwise would be evident at $24 \mathrm{~h}$.

Cx26 junctional channels, but not hemi-channels, have a significant role in the propagation of signalling events leading to DNA damage in bystander cells. To distinguish the roles of Cx26 junctional channels and hemi-channels in the propagation of signalling events leading to DNA damage in the bystander cells, AG1522 bystander cells were cocultured with irradiated HeLa Cx26 cells in the presence or absence of the gap junction inhibitor AGA or the hemi-channel inhibitor $\mathrm{La}^{3+}$ (Figure 2). A 24-h coculture period in the presence of $\mathrm{La}^{3+}$ had essentially no effect on bystander micronucleus formation $(P<0.01)$, but incubation of the cells in the presence of AGA inhibited the bystander induction of DNA damage (Figure 2). That is, inhibition of the junctional channels and not the hemi-channels eliminated the effect of Cx26 expression on micronucleus formation in bystander cells. These results show that $\mathrm{Cx} 26$ likely mediates the bystander response through its role in forming junctional channels. Compared with the data in Figure 1B, where a bystander effect was detected in AG1522 cells cocultured for $24 \mathrm{~h}$ with parental HeLa cells devoid of functional $\mathrm{Cx}$, these results suggest that communication through gap junctions supersedes the effect of events propagated by extracellular secretion.

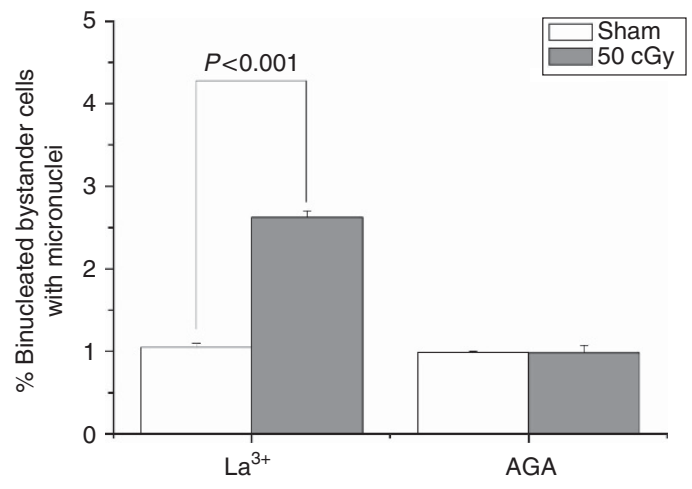

Figure 2. The role of connexin26 channels in the propagation of harmful effects of ionising radiation. Micronucleus formation in bystander AG1522 cells that were cocultured for $24 \mathrm{~h}$ with $\alpha$-particleirradiated or sham-treated contiguous HeLa cells expressing Cx26 in the presence of non-toxic concentrations $(50 \mu \mathrm{m})$ of the gap junction inhibitor AGA or the hemi-channel inhibitor $\mathrm{La}^{3+}$.

COX-2 knockdown attenuates radiation bystander effects mediated by gap junctions and diffusible factor(s). To investigate whether COX-2 signalling has a modulating role in gap junction-mediated bystander effects, we knocked down COX-2 level in HeLa parental, HeLa Cx26 and HeLa Cx32 cells by shRNA constructs that target different regions of the COX-2 mRNA sequence. The clones with the best knockdown efficiency were used in the experiments (Supplementary Figure 1). Similar to the results in Figure 1, micronucleus formation in bystander AG1522 cells cocultured with irradiated HeLa parental cells for $24 \mathrm{~h}$ but not $6 \mathrm{~h}$ was increased $(P<0.01)$ (Figure $3 \mathrm{~A}$ and $\mathrm{B})$; the knockdown of COX-2 prevented this increase (Figure 3B), suggesting that irradiation induces a COX-2-mediated pathway leading to the component of bystander toxicity that is not mediated by gap junctions. Strikingly, knockdown of COX-2 in HeLa Cx26 cells also attenuated the bystander effect detected at both $6 \mathrm{~h}$ and $24 \mathrm{~h}$ of coculture (Figure 3C and D). Except for the results obtained with cells from clone 5 where the increase in micronucleus formation was still significant at $6 \mathrm{~h}(P<0.015$ vs $P<0.001$ in respective control) (Figure $3 \mathrm{C}$ ), the trend towards an increase in micronucleus formation in the other clone and at $24 \mathrm{~h}$ in clone 5 was not significant when COX-2 basal level was decreased in the irradiated cells (Figure 3C and D). On the other hand, knockdown of COX-2 in HeLa cells expressing Cx32 did not alter the level of DNA damage in the form of micronuclei in bystander AG1522 cells examined at either $6 \mathrm{~h}$ or $24 \mathrm{~h}$ following coculture (Figure $3 \mathrm{E}$ and F). There were no significant differences in micronucleus 

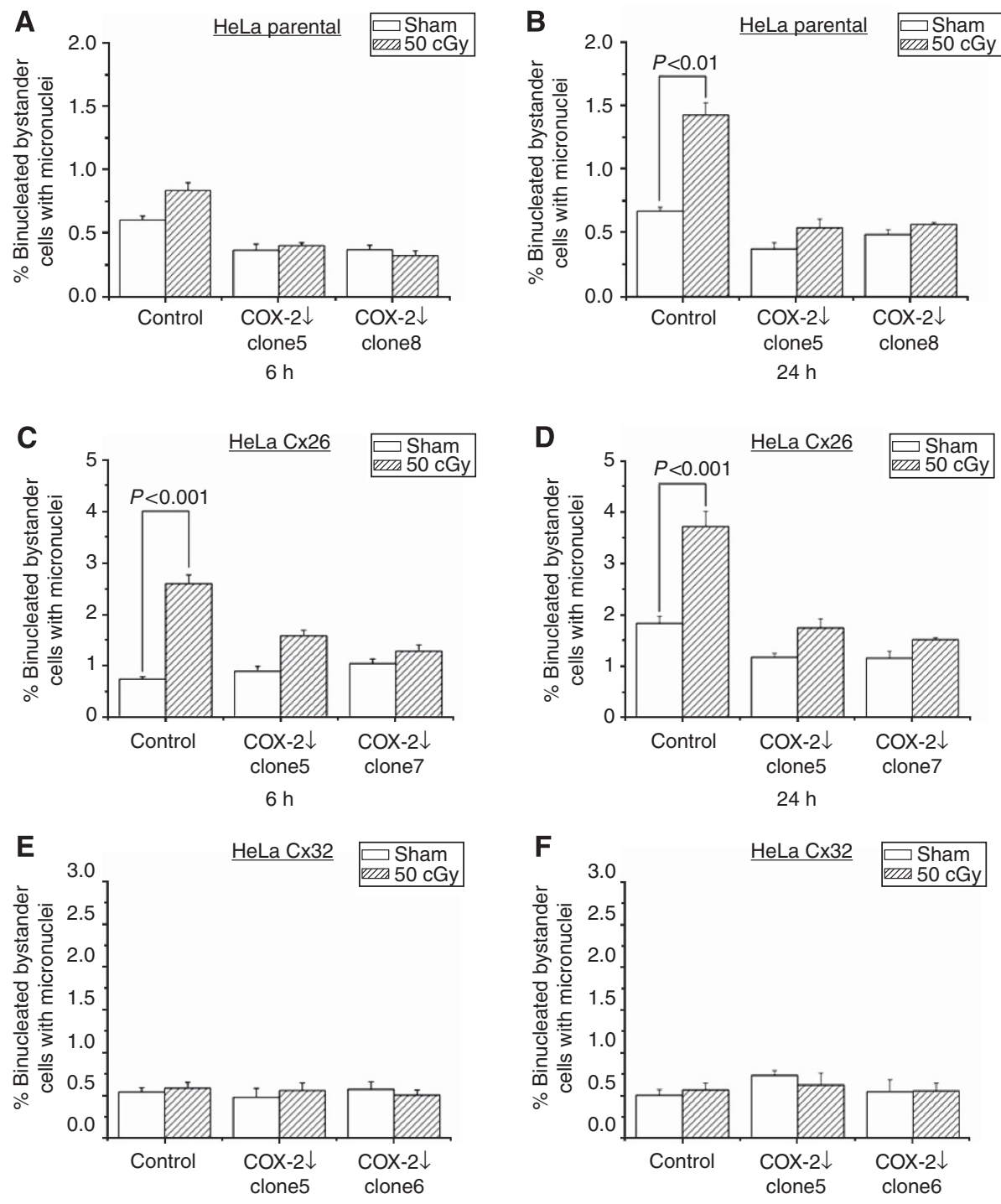

$24 \mathrm{~h}$

Figure 3. The effect of COX-2 on radiation-induced bystander effects mediated by connexin channels. Micronucleus formation in bystander normal human AG1522 fibroblasts cocultured with contiguous HeLa cells with normal (control) or knockdown levels of COX-2. HeLa parental cells (A and $\mathbf{B})$ and HeLa cells expressing Cx26 (C and $\mathbf{D})$ or $\mathbf{C} \times 32$ (E and $\mathbf{F})$ with normal or knockdown levels of COX-2 were exposed to a mean absorbed dose of $50 \mathrm{cGy}$ from $3.7 \mathrm{MeV} \alpha$ particles or were sham-treated. Within $5 \mathrm{~min}$ after exposure, they were intimately cocultured with AG1522 fibroblasts for $6 \mathrm{~h}$ (left panels) or $24 \mathrm{~h}$ (right panels). After coculture, the bystander cells were harvested and subjected to the micronucleus formation assay.

formation in AG1522 cells cocultured with irradiated or sham-treated HeLa Cx32 cells originating from either of the 2 clones used.

COX-2 knockdown delays cellular coupling via Cx26 channels. To test the effect of COX-2 knockdown on cellular coupling, HeLa Cx26 cells with normal or knockdown COX-2 were loaded with CellTracker Orange CMTMR fluorescent dye (Molecular Probes, Eugene, OR, USA) that remains tightly bound to cells. In addition, the cells were loaded with Calcein AM (Molecular Probes). Once loaded into the cell, this dye can migrate to adjacent cells through gap junctions only. After loading, the cultures were trypsinised and the cells were replated with unloaded AG1522 bystander cells at a respective ratio of 1:99 and seeded at high density. At different times thereafter, the cells were examined with a fluorescent microscope. The data in Figure 4 (top row) show that CellTracker Orange-loaded HeLa Cx26 cells with normal COX-2 establish junctional communication with AG1522 cells within $2 \mathrm{~h}$ of coculture, as illustrated by the transfer of Calcein AM dye. When HeLa Cx26 with COX-2 knockdown were cocultured with AG1522 cells, functional coupling also occurred; however, it was attenuated and delayed (Figure 4, middle and bottom rows). At $2 \mathrm{~h}$ and $3.5 \mathrm{~h}$ of coculture, it was only $\sim 15 \%$ and $35 \%$, respectively, of the coupling observed at $2 \mathrm{~h}$ in HeLa Cx26 cells with normal COX-2 level.

COX-2 knockdown affects the interaction of $\mathrm{Cx} 26$ with the mitogen-activated protein kinase kinase kinase (MAPKKK) MLT. To gain a greater understanding of the crosstalk between COX-2 and gap junction coupling, we characterised Cx26interacting proteins in the plasma membrane of HeLa cells with normal or knockdown COX-2 levels. Cholesterol-rich domains of the plasma membrane were isolated, and proteins that coimmunoprecipitated with Cx26 were purified and identified by liquid chromatography-mass spectrometry (LC-MS). Among other proteins, MLTK was found to exist in complex with Cx26 in cells with normal COX-2 level. MLTK is a member of the MAPKKK 
A

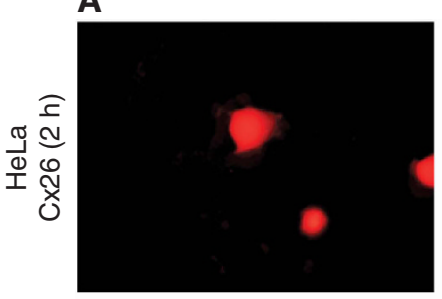

B
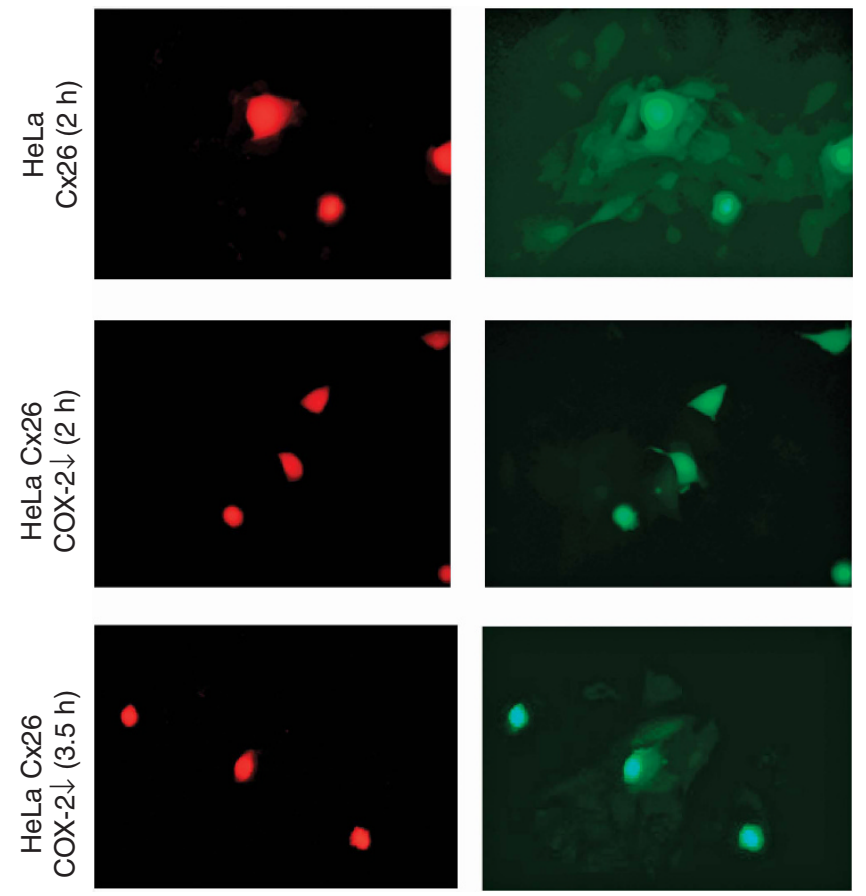

Figure 4. The effect of COX-2 knockdown on junctional communication between HeLa Cx26 cells and AG1522 fibroblasts. HeLa Cx26 cells loaded with Calcein AM (green fluorescence) and CellTracker Orange (red fluorescence) were mixed with non-loaded AG1522 cells at a respective ratio of $1: 99$. Cells were microscopically examined with Texas Red (A) or FITC filters (B). (A) Representative field showing the loaded cells present in the coculture. (B) Same field in (A) showing Calcein AM dye transfer that occurs between HeLa Cx26 cells with normal or knockdown level of COX-2 and AG1522 fibroblasts at $2 \mathrm{~h}$ and $3.5 \mathrm{~h}$ after coculture.

family and is upstream of $\mathrm{p} 38^{\mathrm{MAPK}}$ (Gotoh et al, 2001) that regulates the expression of COX-2 (Zhou et al, 2005) and several radiation-induced responses, including bystander effects (Azzam et al, 2002; Asur et al, 2010). Further, phosphorylation of connexin by MAPK affects its interaction with other proteins (Cameron et al, 2003). The knockdown of COX-2 reduced the interaction of Cx26 with MLTK by $\sim 8$-fold. The immunoprecipitation/immunoblotting results in Figure 5 confirm the LC-MS results and show that knockdown of COX-2 drastically attenuated the Cx26-MLTK interaction. However, this reduced interaction did not affect the activation of ERK1/2, p38 ${ }^{\mathrm{MAPK}}$ and NF- $\kappa \mathrm{B}$ in HeLa Cx26 cells exposed to $\alpha$ particles (Figure 6). Interestingly though, in shamtreated cells, the basal levels of ERK1/2, p-ERK1/2, p38 ${ }^{\mathrm{MAPK}}$, $\mathrm{p}-\mathrm{p} 38^{\mathrm{MAPK}}$ and $\mathrm{p}-\mathrm{NF}-\kappa \mathrm{B}$ were lower in COX-2 knockdown cells compared with cells with a normal level of COX-2 $(0.73 \pm 0.31$, $0.55 \pm 0.01,0.61 \pm 0.14,0.36 \pm 0.22$, and $0.6 \pm 0.02$, respectively).

COX-2 knockdown promotes genomic instability in progeny of normal bystander cells cocultured with cancer cells expressing Cx26. The results in Figure 3 indicate that knockdown of COX-2 attenuates stressful effects in normal bystander cells examined within hours of coculture with irradiated cancer cells. To investigate the long-term outcome of the bystander effect, we evaluated spontaneous micronucleus formation in progeny of the bystander cells. Bystander AG1522 cells cocultured with irradiated or sham-treated HeLa Cx26 cells with normal or knockdown COX2 levels were harvested after $6 \mathrm{~h}$ of coculture. They were then propagated for 20 population doublings and examined for micronucleus formation. There was no difference in micronucleus formation in progeny of AG1522 cells that were previously
Control

$\operatorname{cox}-2 \downarrow$

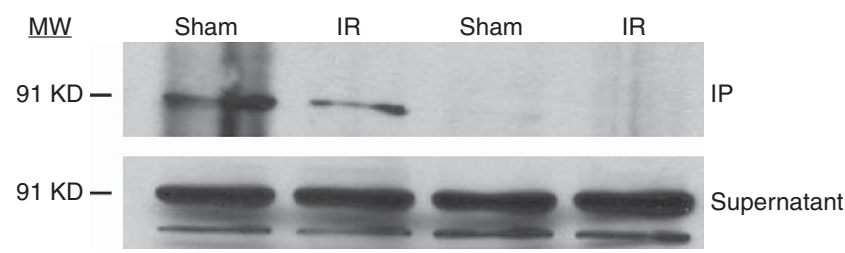

Figure 5. The effect of COX-2 knockdown on the plasma membrane interaction of connexin26 with Mitogen-Activated Protein Kinase Kinase Kinase (MAPKKK). Physical interaction of Cx26 with MAPKKK MLK in cholesterol-rich domains of the plasma membrane of irradiated (4 Gy from ${ }^{137} \mathrm{Cs} \gamma$ rays) or sham-treated HeLa Cx26 cells with normal or COX-2 knockdown. Western blot analyses of MAPKKK MLK in the supernatant and pelleted fraction after immunoprecipitation with antiCx26 antibody.

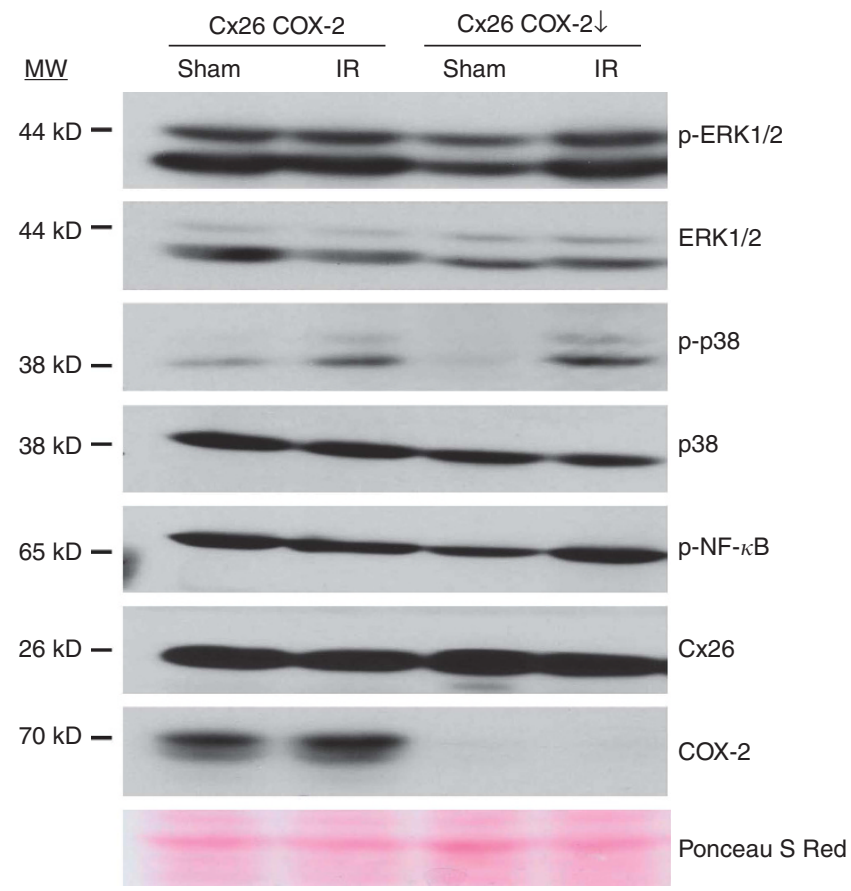

Figure 6. Effect of COX-2 knockdown on the levels of stressresponsive proteins in sham-treated and irradiated HeLa Cx26 cells. Western blot analyses of COX-2 regulatory proteins in HeLa Cx26 cells with normal or COX-2 knockdown at $3 \mathrm{~h}$ after irradiation with a mean absorbed dose of $50 \mathrm{cGy}$ from $3.7 \mathrm{MeV} \alpha$-particles. Staining with Ponceau S Red was used as loading control.

cocultured with irradiated or sham-treated HeLa Cx26 cells with normal COX-2 level $(P=0.36)$ (Figure 7$)$. In contrast, progeny of bystander cells that were cocultured with irradiated HeLa Cx26 cells with COX-2 knockdown, which showed a short-term attenuated bystander effect (Figure 3C), exhibited an enhanced genomic instability reflected in a greater fraction of progeny cells harbouring micronuclei $(P<0.01)$ (Figure 7$)$.

\section{DISCUSSION}

Our micronucleus formation results (Figures 1-3) indicate that several pathways mediate the spread of stressful effects from irradiated cells to neighbouring bystander cells, which results in induction of DNA damage in the bystander cells. These effects, whether mediated by junctional communication or extracellular 


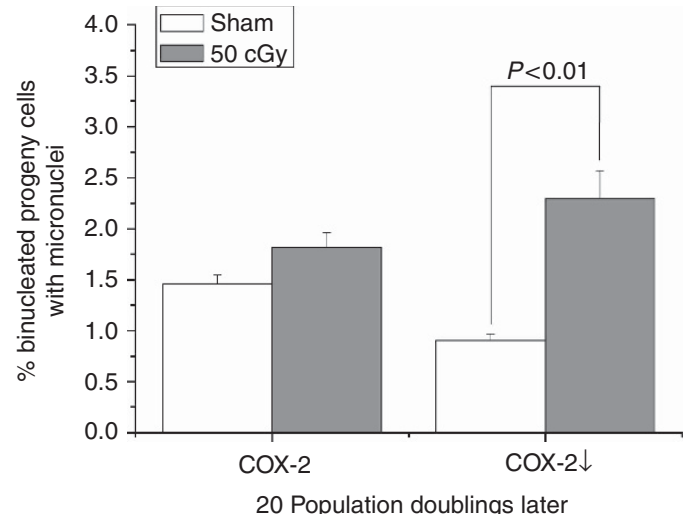

Figure 7. Effect of COX-2 knockdown in irradiated HeLa Cx26 on delayed changes induced in progeny of bystander normal cells.

Micronucleus formation in progeny of isolated bystander AG1522 cells that were previously cocultured with $\alpha$-particle-irradiated or shamtreated HeLa Cx26 cells with normal or COX-2 knockdown for $6 \mathrm{~h}$.

secretion, are significantly modulated by COX-2 (Figures 1-3). Clearly, the expression of harmful bystander effects impacts the long-term outcome of therapeutic irradiation.

Not unexpectedly, the effects propagated through gap junctions superseded those that are disseminated through extracellular secretion (Figure 1), as this type of communication surmounts the barrier presented by the intervening plasma membrane. These results build upon a wealth of evidence showing that radiation injury extends from the irradiated to non-irradiated cells in an exposed cell population. Specifically, our data reveal the novel finding that the magnitude/nature of the effects communicated from irradiated tumour to normal cells is dependent on the permeability of the channels that couple these cells together (Figures 1 and 3). Whereas inducible expression of Cx26 channels propagated radiation effects that resulted in excess DNA damage in bystander cells, intercellular communication through $\mathrm{Cx} 32$ channel helped prevent the expression of such an effect (Figure 1A and B). Previous studies have shown that depending on their isoform composition connexin channels can discriminate between highly similar second messengers (e.g., cAMP and cGMP, and among inositol triphosphates) (Harris, 2001). The mitigating effect of Cx32 channels may be due to enhanced or reduced permeability to certain signalling molecules. Rescue factors may be also communicated from the bystander to the irradiated cells, which would counteract the propagation of signalling molecules that lead to harmful bystander responses. However, this does not preclude a role for the involvement of $\mathrm{Cx} 32$ effects that are independent of junctional communication, including the secretion of protective molecules. Specifically, experiments wherein HeLa Cx26 cells or HeLa Cx32 cells are cocultured with AG1522 cells in which Cx26 or $\mathrm{Cx} 32$ are knocked down would shed light on the specificity of these Cx in RIBE and the ways by which they mediate their effects.

Similar to gap junctions, hemi-channels also affect physiological processes that impact cell survival (Harris, 2001). Our results support the concept that the harmful bystander effects propagated by irradiated HeLa cells expressing Cx26 are due to the role of this protein in the formation of junctional channels and not hemichannels. Incubation of irradiated and contiguous bystander cells with the gap junction inhibitor AGA (Gaillard et al, 2009), and not the hemi-channel inhibitor $\mathrm{La}^{3+}(50 \mu \mathrm{M})$, inhibited the induction of DNA damage in bystander cells (Figure 2). Clearly, additional experiments investigating higher concentrations of $\mathrm{La}^{3+}$ (Wang et al, 2013) may yield different outcomes; however, interpretations of the results may be complicated owing to toxic effects of the higher concentrations. Furthermore, the effects of AGA on bystander effects induced in AG1522 cells cocultured with irradiated HeLa parental cells (deficient in gap junction communication) would be informative; lack of an effect on the magnitude of the bystander response would support that inhibition of micronucleus induction in bystander AG1522 cells cocultured with irradiated HeLa Cx26 cells in the presence of AGA was due to inhibition of junctional communication. Prominently, our results have shown that the effects mediated by inducing Cx26 expression in HeLa cells were greatly affected by COX-2 level. COX-2 knockdown significantly reduced the extent of harmful bystander effects being propagated from irradiated HeLa Cx26 cells (Figure 3C and D).

Previous studies had shown that COX-2 is an important mediator of bystander effects (Zhou et al, 2005). The results in Figure 3 support these findings and show that COX-2 modulates bystander effects mediated by both extracellular secretion and gap junctions. In irradiated HeLa parental cells devoid of functional Cx, downregulation of COX-2 abrogated the expression of harmful bystander effects observed at $24 \mathrm{~h}$ of coculture (Figure $3 \mathrm{~A}$ and $\mathrm{B}$ ). In irradiated HeLa cells expressing Cx26, COX-2 knockdown greatly affected the induction of micronuclei in bystander cells. Interestingly, the basal level of COX-2 was decreased in HeLa cells induced to express Cx26 (Supplementary Figure 2), and COX-2 knockdown affected the expression of mediators of the bystander effect, namely p $38^{\mathrm{MAPK}}$ and NF- $\kappa \mathrm{B}$ (Figure 6).

In contrast to HeLa Cx26 cells, knockdown of COX-2 in HeLa Cx32 cells had no modulating effect on bystander responses assessed at $6 \mathrm{~h}$ or $24 \mathrm{~h}$ of coculture; there was no induction of micronuclei in bystander AG1522 cells cocultured with irradiated HeLa Cx32 cells expressing normal or reduced levels of COX-2 (Figure 3). The absence of an effect suggests that the rescue role of Cx32 channels is independent of COX-2, or the effects mediated by these channels block COX-2 effect(s) that lead to micronucleus formation in bystander cells.

Our results also showed that COX-2 is critical in mediating nontargeted effects when modulated at the level of the bystander cells. Bystander HeLa parental cells with COX-2 knockdown that shared medium with cocultured irradiated HeLa parental cells with normal COX-2 expression did not exhibit an increase in micronucleus formation compared with the respective control (Supplementary Figure 3).

Whereas junctional propagation of radiation-induced effects that enhance cell killing of tumour cells increases the therapeutic gain (Autsavapromporn et al, 2011), propagation of effects that enhance genomic instability in progeny of normal bystander cells may account for secondary harmful effects. Although knockdown of COX-2 in irradiated HeLa Cx26 cells (clone 7) attenuated harmful effects assessed at $6 \mathrm{~h}$ or $24 \mathrm{~h}$ in AG1522 bystander cells, the progeny of the bystanders exhibited significant enhancement in spontaneous micronucleus formation 20 population doublings later (Figure 7). In contrast, the progeny of AG1522 bystander cells that were cocultured with irradiated HeLa Cx26 cells with normal COX-2 level did not show such a persistent harmful effect (Figure 7). Likely, the level of stress communicated from the latter cells to the bystanders shortly after irradiation contributed to death of the affected bystanders or permanently inhibited their proliferation. On the other hand, the emergence of a significant fraction of cells harbouring micronuclei among the progeny of bystanders that were apparently unaffected shortly after coculture (i.e., AG1522 cells cocultured with irradiated HeLa Cx26 knockdown cells) is consistent with accumulating changes in these cells. Our earlier studies had shown that progeny of bystander cells from cultures exposed to very low mean absorbed doses of densely ionising radiations experience perturbations in oxidative metabolism manifested in decreased antioxidant potential, decreased aconitase activity and increased level of oxidised proteins (Buonanno et al, 2011).

Connexins are known to interact in the plasma membrane with other junctional proteins, enzymes, structural proteins and other molecules (Harris, 2001). Our study shows that these interactions are 
affected by COX-2 knockdown. Specifically, the physical interaction of Cx26 with the MAPKKK MLT in the plasma membrane was drastically reduced (Figure 5). The MAPKKK participates in many functions, including DNA damage checkpoints and apoptosis. Importantly, it is implicated in the activation of $\mathrm{p} 38^{\mathrm{MAPK}}$ that we and others have shown to be involved in ionising RIBE (Azzam et al, 2002; Asur et al, 2010; Guo et al, 2013). Significantly, p38 $8^{\mathrm{MAPK}}$ regulates COX-2 activity (Hei, 2006), and the phosphorylation of connexin by MAPK or other kinases regulates gap junction assembly and the role of $\mathrm{Cx}$ in proliferation and in other physiological pathways (Solan and Lampe, 2005). It is attractive therefore to speculate that the decreased interaction of Cx26 with MAPKKK may be a critical link in the crosstalk between connexin and COX-2.

In conclusion, this study shows that multiple mechanisms participate in the propagation of stressful effects from irradiated to bystander cells. When cells are able to communicate by direct and indirect modes, the most efficient mode (i.e., junctional communication) predominates (Figures 1 and 2). Whereas different signalling molecules may be communicated via extracellular secretion or junctional communication, a common modulating effect seems to involve COX-2 activity: COX-2 modulates bystander responses at the level of both the irradiated and bystander cells (Figures 1-3, Supplementary Figure 3). Importantly, functional coupling between HeLa Cx26 cells and AG1522 fibroblasts is significantly delayed upon COX-2 knockdown (Figure 4); whether this is due to altered phosphorylation of $\mathrm{Cx} 26$ remains to be investigated. Characterising the temporal interactions of $\mathrm{Cx}$ with partner kinases/phosphatases in control, irradiated and bystander cells with normal or COX-2 knockdown levels will likely provide further clues to the pathways that regulate intercellular communications by gap junctions. Such studies will likely reveal that different protein interactions occur with the various $\mathrm{Cx}$ as a function of time after treatment. Together, these studies, and their expansion to intact tissues/organs, will contribute to a greater understanding of nontargeted responses in the entire biological system and by corollary of the debilitating pathologies that arise after cancer radiotherapy.

\section{ACKNOWLEDGEMENTS}

We thank Drs Tong Liu, Xiangwen Chen-Deutsch and Jason Domogauer for their critical input in experiments and Professor Andrew L. Harris for his gift of HeLa cells. This work was supported by grant CA049062 from the NIH.

\section{REFERENCES}

Asur R, Balasubramaniam M, Marples B, Thomas RA, Tucker JD (2010) Involvement of MAPK proteins in bystander effects induced by chemicals and ionizing radiation. Mutat Res 686(1-2): 15-29.

Autsavapromporn N, de Toledo SM, Jay-Gerin JP, Harris AL, Azzam EI (2013) Human cell responses to ionizing radiation are differentially affected by the expressed connexins. J Radiat Res (Tokyo) 54(2): 251-259.

Autsavapromporn N, de Toledo SM, Little JB, Jay-Gerin J-P, Harris AL, Azzam EI (2011) The role of gap junction communication and oxidative stress in the propagation of toxic effects among high-dose $\alpha$-particleirradiated human cells. Radiat Res 175(3): 347-357.

Azzam EI, de Toledo SM, Gooding T, Little JB (1998) Intercellular communication is involved in the bystander regulation of gene expression in human cells exposed to very low fluences of alpha particles. Radiat Res 150: 497-504.

Azzam EI, de Toledo SM, Little JB (2001) Direct evidence for the participation of gap-junction mediated intercellular communication in the transmission of damage signals from alpha-particle irradiated to non-irradiated cells. Proc Natl Acad Sci USA 98(2): 473-478.
Azzam EI, de Toledo SM, Little JB (2003) Expression of connexin43 is highly sensitive to ionizing radiation and environmental stresses. Cancer Res 63(21): 7128-7135.

Azzam EI, de Toledo SM, Spitz DR, Little JB (2002) Oxidative metabolism modulates signal transduction and micronucleus formation in bystander cells from alpha-particle-irradiated normal human fibroblast cultures. Cancer Res 62(19): 5436-5442.

Buonanno M, De Toledo SM, Pain D, Azzam EI (2011) Long-term consequences of radiation-induced bystander effects depend on radiation quality and dose and correlate with oxidative stress. Radiat Res 175(4): 405-415.

Cameron SJ, Malik S, Akaike M, Lerner-Marmarosh N, Yan C, Lee JD, Abe J, Yang J (2003) Regulation of epidermal growth factor-induced connexin 43 gap junction communication by big mitogen-activated protein kinase1/ERK5 but not ERK1/2 kinase activation. J Biol Chem 278(20): 18682-18688.

Gaillard S, Pusset D, de Toledo SM, Fromm M, Azzam EI (2009) Propagation distance of the alpha-particle-induced bystander effect: the role of nuclear traversal and gap junction communication. Radiat Res 171(5): 513-520.

Goodenough DA, Revel JP (1970) A fine structural analysis of intercellular junctions in the mouse liver. J Cell Biol 45(2): 272-290.

Gotoh I, Adachi M, Nishida E (2001) Identification and characterization of a novel MAP kinase kinase kinase, MLTK. J Biol Chem 276(6): 4276-4286.

Guo X, Sun J, Bian P, Chen L, Zhan F, Wang J, Xu A, Wang Y, Hei TK, Wu L (2013) Radiation-induced bystander signaling from somatic cells to germ cells in Caenorhabditis elegans. Radiat Res 180(3): 268-275.

Harris AL (2001) Emerging issues of connexin channels: biophysics fills the gap. Q Rev Biophys 34(3): 325-472.

Hei TK (2006) Cyclooxygenase-2 as a signaling molecule in radiation-induced bystander effect. Mol Carcinog 45(6): 455-460.

Jensen R, Glazer PM (2004) Cell-interdependent cisplatin killing by $\mathrm{Ku} /$ DNA-dependent protein kinase signaling transduced through gap junctions. Proc Natl Acad Sci USA 101(16): 6134-6139.

Koreen IV, Elsayed WA, Liu YJ, Harris AL (2004) Tetracycline-regulated expression enables purification and functional analysis of recombinant connexin channels from mammalian cells. Biochem J 383(Pt 1): 111-119.

Mothersill C, Seymour CB (2004) Radiation-induced bystander effects-implications for cancer. Nat Rev Cancer 4(2): 158-164.

Nagasawa H, Little JB (1992) Induction of sister chromatid exchanges by extremely low doses of $\alpha$-particles. Cancer Res 52: 6394-63396.

Prise KM, O’Sullivan JM (2009) Radiation-induced bystander signalling in cancer therapy. Nat Rev Cancer 9(5): 351-360.

Solan JL, Lampe PD (2005) Connexin phosphorylation as a regulatory event linked to gap junction channel assembly. Biochim Biophys Acta 1711(2): 154-163.

Stasinopoulos I, Shah T, Penet MF, Krishnamachary B, Bhujwalla ZM (2013) COX-2 in cancer: Gordian knot or Achilles heel? Front Pharmacol 4: 34.

Subbaramaiah K, Chung WJ, Dannenberg AJ (1998) Ceramide regulates the transcription of cyclooxygenase-2. Evidence for involvement of extracellular signal-regulated kinase/c-Jun N-terminal kinase and p38 mitogen-activated protein kinase pathways. J Biol Chem 273(49): 32943-32949.

Wang N, De Bock M, Decrock E, Bol M, Gadicherla A, Vinken M, Rogiers V, Bukauskas FF, Bultynck G, Leybaert L (2013) Paracrine signaling through plasma membrane hemichannels. Biochim Biophys Acta 1828(1): 35-50.

Willecke K, Eiberger J, Degen J, Eckardt D, Romualdi A, Guldenagel M, Deutsch U, Sohl G (2002) Structural and functional diversity of connexin genes in the mouse and human genome. Biol Chem 383(5): 725-737.

Zhou H, Ivanov VN, Gillespie J, Geard CR, Amundson SA, Brenner DJ, Yu Z, Lieberman HB, Hei TK (2005) Mechanism of radiation-induced bystander effect: role of the cyclooxygenase-2 signaling pathway. Proc Natl Acad Sci USA 102(41): 14641-14646.

Zhou HN, Suzuki M, Randers-Pehrson R, Chen G, Trosko J, Vannais D, Waldren CA, Hall EJ, Hei TK (2001) Radiation risk at low doses may be greater than we thought. Proc Natl Acad Sci USA 98(25): 14410-14415.

This work is published under the standard license to publish agreement. After 12 months the work will become freely available and the license terms will switch to a Creative Commons AttributionNonCommercial-Share Alike 3.0 Unported License. 